\title{
Autism-Spectrum Quotient Is Associated with Observational Skill Acquisition in Healthy Young Adults
}

\author{
Yoichiro Sato $^{1 *}$, Kento Kageyama ${ }^{2}$ \\ ${ }^{1}$ Department of Physical Therapy, Faculty of Health Sciences, Hokkaido University of Science, Sapporo, Japan \\ ${ }^{2}$ Department of Rehabilitation, Asahikawa Medical University Hospital, Asahikawa, Japan \\ Email: *sato-y@hus.ac.jp
}

How to cite this paper: Sato, Y. and Kageyama, K. (2020) Autism-Spectrum Quotient Is Associated with Observational Skill Acquisition in Healthy Young Adults. Journal of Behavioral and Brain Science, 10, 179-190.

https://doi.org/10.4236/jbbs.2020.104011

Received: January 30, 2020

Accepted: April 18, 2020

Published: April 21, 2020

Copyright ( 2020 by author(s) and Scientific Research Publishing Inc. This work is licensed under the Creative Commons Attribution International License (CC BY 4.0).

http://creativecommons.org/licenses/by/4.0/ (c) (i) Open Access

\begin{abstract}
Although observational motor learning is one method of skill acquisition, this type of motor learning is not equally effective for all individuals. To clarify factors associated with the effectiveness of motor learning, we examined the association between model-observational skill acquisition and the Autism-Spectrum Quotient $(\mathrm{AQ})$, which is reportedly associated with motor learning via visual information. Twenty healthy adults performed the Kendama task. The participants practiced under three conditions: using their own methods (self), following observation of model actions (model-observation), and following observation of their own actions (self-observation). Measurement trials were performed 20 times prior to self-practice sessions and after each practice session. Success ratios were calculated for each measurement trial. All participants completed the AQ. The difference in success ratios for measurement sessions following practices between the self and model-observation conditions was significantly negatively correlated with AQ scores. Individuals with low AQ values can more easily acquire skills via model-observational motor learning than those with relatively higher $A Q$ values.
\end{abstract}

\section{Keywords}

Observational Motor Learning, Autism-Spectrum Quotient, Motor Imagery, Healthy Individual

\section{Introduction}

Skills can be acquired via motor learning. Although there are various methods of motor learning [1], these methods are not equally effective for all individuals. 
Observational motor learning is one method of motor learning; it involves physical practice following observation of self-actions or actions of another [2] [3] [4]. This type of motor learning is applied not only in sport-related scenarios [5], but also during balance training following stroke [6] [7]. Since direct skill acquisition may be difficult for athletes or patients undergoing rehabilitation, instructors and therapists must determine the type of motor learning most suited to each patient (e.g., model-observational motor learning). Model-observational motor learning is one of the effective methods for skill acquisition [8]. Hence, a specialized tool is needed to determine whether model-observational motor learning is appropriate.

While observing another's actions, individuals collate the characteristics of the observed action with internal models [9] [10] or motor representations in the brain [11]. Previous researchers have suggested that, while practicing the action, individuals correct their movement based on the collation via physical practice [12]. Previous studies have reported that this correction is associated with cerebellar activity [10] [12]. Among the types of motor learning dependent on the cerebellum, motor learning via visual information is impaired in children with autism spectrum disorder (ASD), relative to that in typically developing children [13]. ASD is characterized by impairments in social and communication skills, corresponding with repetitive, stereotyped behaviors [13]. Based on the impairment of motor learning in ASD children, we speculate that the capacity for visual observational motor learning would be lower in healthy individuals with high levels of autistic traits than in those with low levels of autistic traits.

Recent studies have verified that autism exists along a spectrum, and the boundaries between typical development and autism remain unclear [14] [15]. Developed by Baron-Cohen et al. [16], the Autism-Spectrum Quotient (AQ) is used to assess levels of autistic traits in healthy individuals. We speculated that the AQ would be useful for selecting participants suited for skill acquisition via observational motor learning, especially following observation of another's actions. Because children with ASD face greater difficulty with motor learning via visual information than typically developing children [13], we hypothesized that the AQ-which can be used to assess continuity between typical development and ASD-would be associated with the capacity for skill acquisition via model-observational motor learning.

Another type of observational motor learning involves the observation of one's own actions (i.e., self-observational motor learning). We speculated that there would be differences in skill acquisition between self-observational motor learning and model-observational motor learning. Skill acquisition via the observation of self-actions should be associated with motor imagery abilities, whereas skill acquisition via model-observational motor learning should be associated with AQ scores. That is, individuals who cannot imagine how their own bodies move should be able to acquire skills via observation of their own actions. Moreover, there are two types of motor imagery: kinesthetic and visual. Kines- 
thetic motor imagery, also known as first-person motor imagery, is associated with learning via somatosensory information obtained during self-performance of an action. In contrast, visual or third-person motor imagery is associated with observational ability [17]. Individuals with low kinesthetic motor imagery ability fail to grasp their own actions. In such cases, skill acquisition can be improved by having participants review their own actions using a video recording. Hence, we hypothesized that skill acquisition via self-observational motor learning would be associated with motor imagery abilities, especially kinesthetic rather than visual abilities, because low kinesthetic imagery ability should be correlated with poor processing of somatosensory information.

Thus, the present study aimed to demonstrate our hypotheses regarding model- and self-observational motor learning, that there is an association between AQ scores and skill acquisition via observation of another's actions, as well as an association between motor imagery and skill acquisition via observation of self-actions.

\section{Methods}

\section{Participants}

Twenty students were enrolled in the present study (10 men and 10 women). All participants were native Japanese speakers with typical development. They were selected randomly from 120 adult students within the department of physical therapy. The mean (standard deviation [SD]) age of the participants was 20.5 (0.5) years. All participants were healthy and had experienced no physical ailments within the preceding year. Inclusion criteria were as follows: righthandedness, familiarity with the Kendama task without significant practice. Prior to the experiments, participants read a description of the basic procedures of the experiments and provided written informed consent. The experimental procedures of this study were approved by the ethics committee of Hokkaido University of Science in Sapporo, Japan (No. 194).

\section{Tasks}

The Tomeken skill of the Kendama task was adopted for the present experiments (Figure 1). As shown in Figure 1(a), the Kendama task involves a sword and a cup. The top of the sword is sharp, whereas the cup contains a hole. Participants were required to perform the Tomeken skill (Figure 1(b) and Figure 1(c)), which involved lifting the cup attached to the string (Figure 1(a)) and inserting the top of the sword into the hole in the cup. The Kendama task was utilized to elucidate the effect of model-observational motor learning on skill acquisition. This task is ideal for representing actual clinical situations, in which whole-body actions are common. Furthermore, the task does not necessarily depend on muscular strength, in contrast to throwing actions. In addition, accidental success is not possible during the Tomeken task, which exhibits a level of difficulty suitable for Kendama novices.

\section{Procedures}

The experimental protocol is shown in Figure 2. After the $1^{\text {st }}$ measurement 


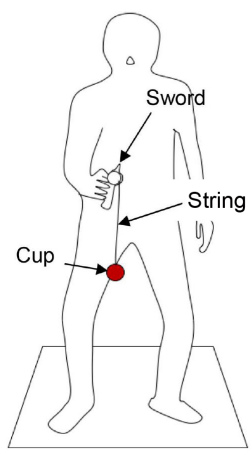

(a)

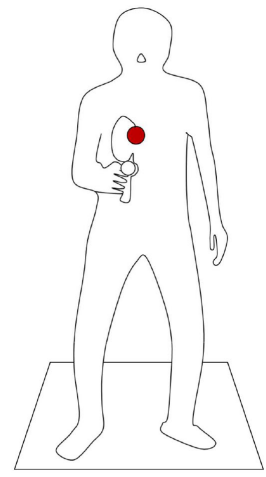

(b)

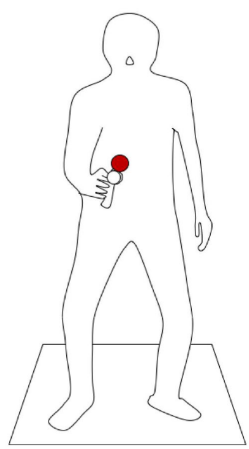

(c)

Figure 1. Whole-body scheme of task action. (a) Starting position; (b) Middle step of the task action, which is to tip up the ball; (c) Finish the task successfully (i.e., the sword is inserted into the hole).

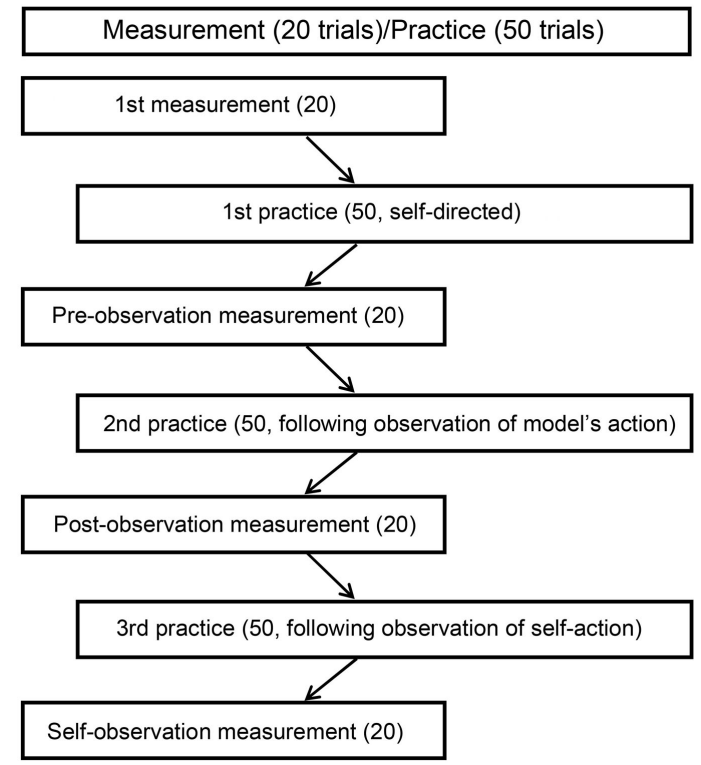

Figure 2. Flowchart of the experiment. The measurements were performed four times, and practice trials were performed three times. After the $1^{\text {st }}$ measurement session, three types of practice were performed prior to each measurement. All participants completed a total of 230 trials.

session, participants were instructed to practice three methods. A basic explanation of the task (i.e., insert the top of the sword into the hole in the cup) was provided to the participants, without explaining how to move the sword or oneself. Each participant was allowed to practice three times prior to the first measurement. Measurements were performed during 20 trials, and the number of successful attempts was recorded during each trial. Next, each participant engaged in 50 self-directed practice trials ( $1^{\text {st }}$ practice). This practice session was designed to distinguish skill acquisition after model-observational motor learning based on physical practice or observation of the model only. After the $1^{\text {st }}$ practice session, a second round of measurements was performed (pre-observation measurements). All participants were then allowed to rest for $1 \mathrm{~min}$, following 
which they watched a video depicting the desired model's actions (URL: https://www.youtube.com/watch?v=zYiygA24Hi4). Although one previous study indicated that representations of another's actions are stronger for real actions than for those observed via video [18], performance of the action may differ among those modeling the action. Therefore, the present study utilized video observation.

The video was edited into four original-speed clips (two whole-body segments and two close-up segments of the acting hand) and one low-speed clip (original speed $\times 0.25)$. Each participant viewed the edited clips three times in succession. The duration of the edited movie was approximately $2 \mathrm{~min}$. Moreover, the explanation regarding the task was erased from the video to ensure that participants could learn the Tomeken task via observation only. After viewing the clips, participants engaged in 50 practice trials attempting to imitate the modeled actions $\left(2^{\text {nd }}\right.$ practice), following which the third measurement session was performed (post-observation measurements). All trials during this session were recorded using a digital video camera (Panasonic, HC-V480M, 60 frames/s). If participants could not acquire the skill via observational motor learning, we assumed that they were unable to perceive how their body moved. Hence, each participant was then allowed to view the movies displaying the modeled actions and his or her own actions in parallel (parallel movie) on a personal computer (PC). This was to ensure that individuals who could not imagine their own actions could intuitively compare self-actions with those of another. There was no audio in the parallel movie. These procedures took approximately 5 min. After viewing the parallel movie, participants engaged in 50 practice trials while attending to differences between their performance and the model's ( $3^{\text {rd }}$ practice), following which the $4^{\text {th }}$ set of measurements was obtained (self-observation measurements). Each participant performed a total of 230 trials, including practice and measurement sessions.

\section{$A Q$ and motor imagery questionnaire}

After completing all trials, each participant completed a paper version of the AQ and the Japanese Movement Imagery Questionnaire-Revised (JMIQ-R).

The AQ consists of 50 items. Each participant selected from among four responses (i.e., definitely agree, slightly agree, slightly disagree, definitely disagree). One point was awarded when participants responded with definitely agree or slightly agree (for inverse items, one point was awarded for responding with definitely disagree or slightly disagree), whereas no points were awarded for other responses. The maximum score of the AQ is 50 points, with higher scores reflecting higher levels of autistic traits [16].

The Movement Imagery Questionnaire (MIQ) was developed by Hall \& Martin [19], and the JMIQ-R was translated by Hasegawa in 2004 [20]. The MIQ has been widely used to investigate motor imagery abilities in patients with stroke [21] and athletes [22]. The JMIQ-R assesses kinesthetic and visual imagery based on the performance of four motions. The JMIQ-R involves the following procedures: 1) the participant poses in the initial posture for each motion, 2) the par- 
ticipant performs each motion only one time, and 3) the participant sits in a chair and imagines each motion performed as in (2). During kinesthetic imagery, participants imagine that they have performed each motion, whereas in visual imagery, they imagine that they have observed their motions from the third-person perspective. Scores on the kinesthetic imagery component range from very difficult to perform ( 1 point) to very easy to perform ( 7 points). Scores on the visual imagery component ranged from very difficult to imagine (1 point) to very easy to imagine ( 7 points). The maximum score on the JMIQ-R is 28 points, with higher scores indicative of better motor imagery ability. Each participant listened to recorded explanations of the JMIQ-R via PC, following which they answered questions regarding visual and kinesthetic imagery.

\section{Data analysis}

In each measurement session, we calculated the success (i.e., insertion of the top of the sword into the hole) ratios across 20 trials for each participant. We calculated differences in the success ratio between post-observation and preobservation measurements (post-pre residual) in order to elucidate how skill acquisition can change via observation of the model's action only. We also calculated the difference between self-observation and post-observation measurements (self-post residual) in order to elucidate how skill acquisition can change via observation of both model- and self-actions.

\section{Statistical analysis}

The Shapiro-Wilk test was used to investigate the normality of each variable. To investigate skill acquisition via observational motor learning in the model condition, Spearman rank correlation coefficients were calculated to examine the association between post-pre residuals and AQ. To investigate the association between skill acquisition via observational motor learning in the self-observation condition, Spearman rank correlation coefficients were calculated to examine the association between self-post residuals and JMIQ-R scores. The level of statistical significance was set at $p<0.05$. All statistical analyses were performed using SPSS version 20.0 (IBM Corporation, Armonk, NY, USA).

\section{Results}

The success ratios for the $1^{\text {st }}$, pre-observation, post-observation, and self-observation measurement sessions were 9.0\%, 15.0\%, 19.0\%, and 22.0\%, respectively (Figure $3)$. Although the ratios increased systematically, the main effect of measurement condition was not significant $\left(F(3,76)=2.027, p=0.117, \eta_{\mathrm{p}}{ }^{2}=0.074\right)$. The median AQ score was 20 (25\% - 75\% tiles: 15.25 - 22). Median scores on the visual and kinesthetic imagery scales were 24 (25\% - 75\% tiles: 21.25 - 28) and $24(25 \%$ - 75\% tiles: 21.25 - 27), respectively (Table 1 ). Spearman rank correlation analysis revealed no significant correlations between AQ and either imagery scale, as well as a significant correlation between motor imagery scales $(r=0.765, p<$ 0.001) (Table 1). The relationship between AQ score and post-pre residuals is shown in Figure 4(a), while that between each JMIQ-R scale and self-post residuals is shown in Figure 4(b). The AQ score significantly decreased as the 


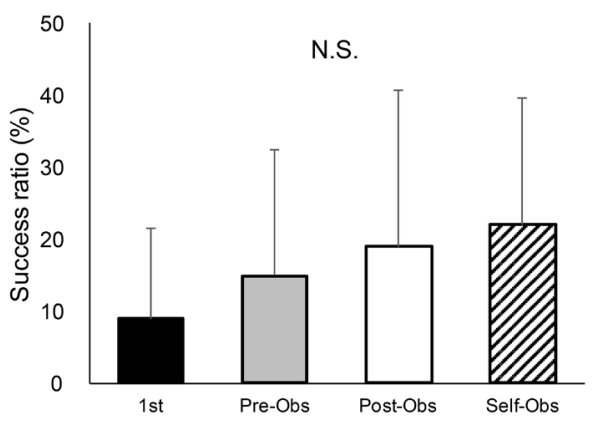

Figure 3. The success ratios in each measurement session. The error bars represent the standard deviation. N.S.: not significant. $1^{\text {st }}$ : first measurement (i.e., no practice); Pre-Obs: pre-observational measurement; Post-Obs: post-observational measurement; Self-Obs: self-observational measurement.

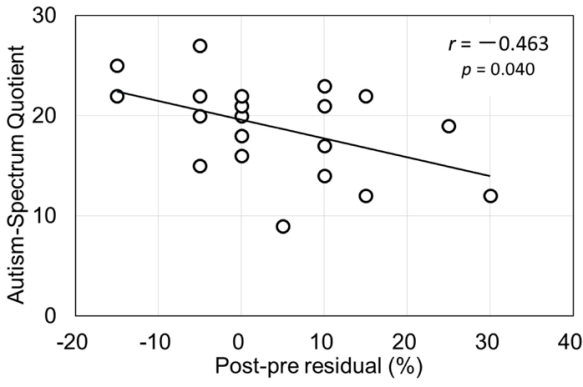

(a)

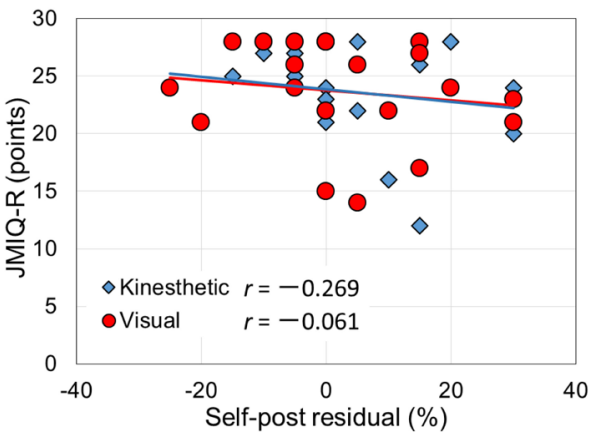

(b)

Figure 4. (a) The relationship between AQ scores and post-pre residuals (post model-observational measurement minus pre-model-observational measurement); (b) The relationship between JMIQ-R scores and self-post residuals (self-observational measurement minus post model-observational measurement). AQ: Autism Spectrum-Quotient; JMIQ-R: Japanese Movement Imagery Questionnaire-Revised.

Table 1. AQ scores, motor imagery scores, and correlation coefficients.

\begin{tabular}{ccccc}
\hline & Median & $(25-75$ tiles $)$ & \multicolumn{2}{c}{ Spearman Coefficient } \\
\cline { 4 - 5 } & & & AQ & Visual \\
\hline AQ & 20 & $(15.25-22)$ & & \\
Visual & 24 & $(21.25-28)$ & -0.117 & $0.765^{* *}$ \\
Kinesthetic & 24 & $(21.25-27)$ & -0.009 &
\end{tabular}

${ }^{* *} p<0.001 ; \mathrm{AQ}:$ Autism-Spectrum Quotient; Visual: Visual imagery; Kinesthetic: Kinesthetic imagery. 
post-pre residual increased $(r=-0.463, p=0.040)$. In contrast, there was no significant correlation between self-post residuals and either JMIQ-R score (kinesthetic imagery: $r=-0.269, p=0.251$; visual imagery: $-0.061, p=0.799$ ).

\section{Discussion}

In the present study, we investigated the association between AQ scores and model-observational skill acquisition, as well as the association between motor imagery and self-observational skill acquisition. We observed a significant correlation between AQ scores and post-pre residuals (post-observation success ratio minus pre-observation success ratio), although neither visual nor kinesthetic motor imagery exhibited a significant correlation with self-post residuals (selfobservation success ratio minus post-observation success ratio).

\section{$A Q$ scores and skill acquisition}

The AQ scores observed in the present study were similar to those reported by Wakabayashi et al. [23], who investigated AQ scores in more than 1,000 college students. Furthermore, there was no significant correlation between AQ scores and motor imagery scales (Table 1), indicating that AQ score does not reflect the same aspects as motor imagery scores. The present AQ scores exhibited a significant negative correlation with post-pre residuals (post-observation success ratio minus pre-observation success ratio) (Figure 4(a)). Moreover, the session condition had no significant main effect on success ratios across all participants. Such findings indicate that success ratios for model-observational motor learning increased as AQ scores decreased, and that healthy individuals with low AQ scores (i.e., low levels of autistic traits [16]) may be more suited to skill acquisition via motor learning than individuals with relatively higher $\mathrm{AQ}$ scores (i.e., high levels of autistic traits). Thus, model-observational motor learning may be effective for individuals with low AQ scores. In contrast, other methods of motor learning, such as self-observational motor learning, may be more appropriate for individuals with high AQ scores. To the best of our knowledge, no studies have investigated the types of motor learning most appropriate for individual patients. Notably, our findings suggest that AQ score can be used to determine which athletes or patients are most suited to model-observational motor learning.

We speculated that AQ scores were associated with model-observational motor learning due to the motor learning characteristics of individuals with ASD. Children with ASD exhibit impairments in the cerebellum, and have greater difficulty with motor learning via visual information than typically developing children [13]. Naturally, observational motor learning relies on visual information. Thus, participants with high AQ scores in the present study may face greater difficulty with motor learning via visual information than those with low AQ scores due to high levels of autistic traits. Future studies should examine the relationships between cerebellar function/volume and observational motor learning in healthy individuals. In addition, impairments in skill acquisition via model-observational motor learning in participants with high AQ scores may be 
associated with mirror neuron activity. Indeed, mirror neurons have been associated with the observation of another's actions [10] [24] [25]. Furthermore, mirror neuron activity occurs when participants observe actions with a specific goal in mind for themselves [26] [27] [28]. Individuals with ASD exhibit low levels of mirror neuron activity [25] [28] [29] and have difficulty grasping the intentions of another's actions [25] [30]. Thus, in the present study, individuals with high levels of autistic traits (i.e., high AQ score) may have had difficulty grasping the intentions of the model's action, and they may not have understood how to practice a multi-joint action such as the Tomeken skill. Taken together, these findings indicate that individuals with high AQ scores may exhibit low mirror neuron activity, inhibiting their ability for physical practice and skill acquisition via model-observational motor learning.

\section{Motor imagery and skill acquisition}

Neither kinesthetic nor visual motor imagery was correlated with self-post residuals (self-observation success ratio minus post-observation success ratio, Figure 4(b)). We hypothesized that kinesthetic imagery, rather than visual imagery, would be associated with skill acquisition via self-observational motor learning. Individuals who can improve skills via self-observational motor learning may not be able to use somatosensory information regarding how their bodies move. However, although the present study demonstrated that the correlation between motor imagery scores and self-post residuals was greater for kinesthetic imagery than for visual imagery, we observed no significant correlations between motor imagery scores and self-post residuals. This discrepancy may be related to the motor imagery scores themselves. The median scores on the visual and kinesthetic imagery subscales were high (Table 1), with some participants attaining perfect scores (28 points). The lack of association may therefore have been due to the relatively small differences among participant scores. Future studies should include a larger number of participants to expand the distribution of motor imagery scores. It is also possible that the upper limit of skill acquisition had been reached by the post-observational practice stage. Indeed, the participants engaged in two 50-trial practice sessions and two 20-trial measurement sessions prior to self-observational practice (i.e., observing one's own actions and the model in parallel), for a total of 140 trials. Thus, participants may have already acquired the Tomeken skill. However, some participants exhibited increases in the success ratio even after self-observational practice (i.e., the mean success was higher for the self-observation session than for the post-observation session, Figure 3), indicating that skill improvement could be observed even after 140 trials. Hence, the experimental setting of this study was valid.

\section{Limitations}

Our results suggested that the relationship between AQ scores and model-observational motor learning may involve impairments in the cerebellum and mirror neuron system. However, we did not utilize brain imaging systems to investigate these associations. Future studies should utilize such systems to determine whether brain function (i.e., the cerebellum or mirror neuron system) is 
associated with observational motor learning. Nonetheless, our findings indicated that AQ scores were significantly correlated with model-observational skill acquisition, suggesting that AQ scores can be used to determine whether individuals are suited to model-observational motor learning. The small sample size of the present study may have been a limitation, but the association between AQ score and model-observational motor learning was statistically significant. This finding may be applicable in clinical situations.

\section{Research implications}

The present findings are useful for coaching athletes and patients regarding strategies to acquire skills in sports and clinical situations. For instance, the model-observation strategy may be effective for individuals with low AQ scores to acquire skills, whereas it may not be effective for individuals with high AQ scores.

\section{Acknowledgements}

The authors thank Editage (www.editage.jp) for their assistance with Englishlanguage editing.

\section{Conflicts of Interest}

The authors declare that they have no conflicts of interest.

\section{References}

[1] McMorris, T. (2004) Acquisition and Performance of Sports Skills. John Wiley \& Sons Ltd., Chichester.

[2] Edward, M.G., Humphreys, G.W. and Castiello, U. (2003) Motor Facilitation Following Action Observation, A Behavioural Study in Prehensile Action. Brain and Cognition, 53, 495-502. https://doi.org/10.1016/S0278-2626(03)00210-0

[3] Maslovat, D., Hodges, N.J., Krigoslon, O.E. and Hamdy, T.C. (2010) Observational Practice Benefits Are Limited to Perceptual Improvements in the Acquisition of a Novel Coordination Skill. Experimental Brain Research, 204, 119-130. https://doi.org/10.1007/s00221-010-2302-7

[4] Nakano, H., Osumi, M., Ueta, K., Kodama, T. and Morioka, S. (2013) Changes in Electroencephalographic Activity during Observation, Preparation, and Execution of a Motor Learning Task. International Journal of Neuroscience, 123, 866-875. https://doi.org/10.3109/00207454.2013.813509

[5] Holmes, P. and Calmels, C. (2008) A Neuroscientific Review of Imager and Observation Use in Sport. Journal of Motor Behavior, 40, 433-445. https://doi.org/10.3200/JMBR.40.5.433-445

[6] Celnik, P., Webster, B., Glasser, D. and Cohen, L.G. (2008) Effects of Action Observation on Physical Training after Stroke. Stroke, 39, 1814-1820. https://doi.org/10.1161/STROKEAHA.107.508184

[7] Hiyamizu, M., Maeoka, H., Matsuo, A. and Morioka, S. (2014) Effects of Self-Action Observation on Standing Balance Learning: A Change of Brain Activity Detected Using Functional Near-Infrared Spectroscopy. NeuroRehabilitaion, 35, 579-585. https://doi.org/10.3233/NRE-141153

[8] Hebert, E. (2018) The Effects of Observing a Learning Model (or Two) on Motor 
Skill Acquisition. Journal of Motor Learning and Development, 6, 4-17. https://doi.org/10.1123/jmld.2016-0037

[9] Flanagan, J.R. and Wing, A.M. (1997) The Role of Internal Models in Motion Planning and Control: Evidence from Grip Force Adjustments during Movements of Hand-Held Loads. Journal of Neuroscience, 17, 1519-1528. https://doi.org/10.1523/JNEUROSCI.17-04-01519.1997

[10] Kawato, M. (1999) Internal Models for Motor Control and Trajectory Planning. Current Opinion in Neurology, 9, 718-727. https://doi.org/10.1016/S0959-4388(99)00028-8

[11] Iacoboni, M., Woods, R.P., Brass, M., Bekkering, H., Mazziotta, J.C. and Rizzolatti, G. (1999) Cortical Mechanisms of Human Imitation. Science, 286, 2526. https://doi.org/10.1126/science.286.5449.2526

[12] Izawa, J., Pekny, S.E., Marko, M.K., Haswell, C.C., Shadmehr, R. and Mostofsky, S.H. (2012) Motor Learning Relies on Integrated Sensory Inputs in ADHD, But Over-Selectively on Proprioception in Autism Spectrum Conditions. Autism Research, 5, 124-36. https://doi.org/10.1002/aur.1222

[13] Marko, M.K., Crocetti, D., Hulst, T., Donchin, O., Shadmehr, R. and Mostofsky, S.H. (2015) Behavioural and Neural Basis of Anomalous Motor Learning in Children with Autism. Brain, 138, 784-797. https://doi.org/10.1093/brain/awu394

[14] Siu, A.L., (US) Preventive Services Task Force (USPSTF), Bibbins-Domingo, K., Grossman, D.C., Baumann, L.C., Davidson, K.W., et al. (2016) Screening for Autism Spectrum Disorder in Young Children: Us Preventive Services Task Force Recommendation Statement. Journal of American Medical Association, 315, 691-696. https://doi.org/10.1001/jama.2016.0018

[15] Hicks, S.D., Rajan, A.T., Wagner, K.E., Barns, S., Carpenter, R.L. and Middleton, F.A. (2018) Validation of a Salivary RNA Test for Childhood Autism Spectrum Disorder. Frontiers in Genetics, 9, 534. https://doi.org/10.3389/fgene.2018.00534

[16] Baron-Cohen, S., Wheelwright, S., Skinner, R., Martin, J. and Clubley, M. (2001) The Autism-Spectrum Quotient (AQ): Evidence from Asperger Syndrome/HighFunctioning Autism, Males and Females, Scientists and Mathematicians. Journal of Autism Developmental Disorder, 31, 5-17. https://doi.org/10.1023/A:1005653411471

[17] Steven, J.A. (2005) Interference Effects Demonstrate Distinct Roles for Visual and Motor Imagery during the Mental Representation of Human Action. Cognition, 95, 329-350. https://doi.org/10.1016/j.cognition.2004.02.008

[18] Caggiano, V., Fogassi, L., Rizzolatti, G., Pomper, J.K., Their, P., Giese, M.A. and Casile, A. (2011) View-Based Encoding of Actions in Mirror Neurons of Area F5 in Macaque Premotor Cortex. Current Biology, 21, 144-148. https://doi.org/10.1016/j.cub.2010.12.022

[19] Hall, C.R. and Martin, K.A. (1997) Measuring Movement Imagery Abilities: A Revision of the Movement Imagery Questionnaire. Journal of Mental Imagery, 21, 143-154. https://doi.org/10.1037/t07979-000

[20] Hasegawa, N. (2004) The Development of a Japanese Version of the Revised Movement Imagery Questionnaire. Imagery Psychological Research, 2, 25-34. (In Japanese)

[21] Sharma, N., Pomeroy, V.M. and Baron, J.C. (2006) Motor Imagery: A Backdoor to the Motor System after Stroke? Stroke, 37, 1941-1952. https://doi.org/10.1161/01.STR.0000226902.43357.fc

[22] Martin, K.A., Moritz, S.E. and Hall, C.R. (1999) Imagery Use in Sport: A Literature 
Review and Applied Model. The Sport Psychologist, 13, 245-268. https://doi.org/10.1123/tsp.13.3.245

[23] Wakabayashi, A., Tojo, Y., BaronCohen, S. and Wheelwright, S. (2004) The Autism-Spectrum Quotient (AQ) Japanese Version: Evidence from High-Functioning Clinical Group and Normal Adults. The Japanese Journal of Psychology, 75, 78-84. (In Japanese) https://doi.org/10.4992/ijpsy.75.78

[24] Gallese, V., Fadiga, L., Fogassi, L. and Rizzolatti, L. (1996) Action Recognition in the Premotor Cortex. Brain, 119, 593-609. https://doi.org/10.1093/brain/119.2.593

[25] Iacoboni, M. and Depretto, M. (2006) The Mirror Neuron System and the Consequences of Its Dysfunction. Nature Reviews Neuroscience, 7, 942-951. https://doi.org/10.1038/nrn2024

[26] Rizzolatti, G. and Craighero, L. (2004) The Mirror-Neuron System. Annual Review of Neuroscience, 27, 169-192. https://doi.org/10.1146/annurev.neuro.27.070203.144230

[27] Rizzolatti, G. and Fogassi, L. (2014) The Mirror Mechanism: Recent Findings and Perspectives. Philosophical Transactions of the Royal Society B, 369, 20130420. https://doi.org/10.1098/rstb.2013.0420

[28] Rizzolatti, G., Cattaneo, L., Fabbri-Destro, M. and Rozzi, S. (2014) Cortical Mechanisms Underlying the Organization of Goal-Directed Actions and Mirror Neuron-Based Action Understanding. Physiological Reviews, 94, 655-706. https://doi.org/10.1152/physrev.00009.2013

[29] Rizzolatti, G. and Fabbri-Destro, M. (2010) Mirror Neurons: From Discovery to Autism. Experimental Brain Research, 200, 223-237. https://doi.org/10.1007/s00221-009-2002-3

[30] Enticott, P.G., Kennedy, H.A., Rinehart, N.J., Tonge, B.J., Bradshaw, J.L., Taffe, J.R., Daskalakis, Z.J. and Fitzgerald, P.B. (2012) Mirror Neuron Activity Associated with Social Impairments But Not Age in Autism Spectrum Disorder. Biological Psychiatry, 71, 427-433. https://doi.org/10.1016/j.biopsych.2011.09.001 